\title{
Symmetry and the Crossing Number for Complete Graphs*
}

\author{
Thomas L. Saaty**
}

(February 26, 1969)

\begin{abstract}
This paper studies the minimum number of intersections of edges in a complete graph on $n$ vertices drawn in the plane. The proofs are first given for $n \leqslant 10$. A theorem on the maximum number of intersections is also given. Geometric representations of these cases are included. Symmetry of the representations is then discussed as it applies to extensions from small values of $n$ to larger values maintaining the minimality of the number of intersections. Based on a symmetry conjecture given in the paper, a proof is given for the general case of the minimum intersection problem.
\end{abstract}

Key words: Complete graphs; intersections, symmetry.

\section{Introduction}

We are concerned with the conjecture that the minimum number of intersections $I_{n}$ of the edges of a complete graph on $n$ vertices drawn in the plane in which two edges intersect in at most one point and two edges with a vertex in common have no intersections, is given by

$$
\begin{aligned}
& I_{n}=\frac{n(n-2)^{2}(n-4)}{64} \text { for } n \text { even } \\
& I_{n}=\frac{(n-1)^{2}(n-3)^{2}}{64} \text { for } n \text { odd. }
\end{aligned}
$$

In a complete graph every vertex is connected to each other vertex by one edge. We shall use $C_{n}$ to denote a complete graph on $n$ vertices and call it minimal (maximal) if it has a minimum (maximum) number of intersections. In reference $[10]^{1}$ a realization scheme to which we shall make frequent reference is given. Based on that construction and on those of related problems, the notion of symmetry for combinatorial problems is introduced and a conjecture in the form of a principle is given.

\section{Proof of Small Cases}

Consider each vertex of the graph $C_{n}$ and its $n-1$ connecting edges (its star) to the remaining vertices. The removal of this vertex and its star, eliminates ail intersections falling on these edges. The result is a complete graph_on $n=1$ vertices. Any other vertex of the complete graph on $n$ vertices has the same or a different number of intersections on its star. In general, if $x_{i}$, $i=1, \ldots, n$ is the number of intersections on the edges connecting the $i$ th vertex then the total number of intersections of $C_{n}$ is $\frac{1}{4} \sum_{i=1}^{n} x_{i}$ since each intersection is counted exactly four times, once for each of the four vertices of the two edges defining the intersection. Thus we have:

\footnotetext{
*An invited paper.

**Present address: Arms Control and Disarmament Agency, Science and Technology Bureau, 2201-C Street, N.W., Washington, D.C. 20451.

${ }^{1}$ Figures in brackets indicate the literature references at the end of this paper.
} 
THEOREM 1: If $\mathrm{x}_{\mathrm{i}}, \mathrm{i}=1, \ldots, \mathrm{n}$ is the number of intersections falling on the star of the $\mathrm{i}$ th vertex of a complete graph $\mathrm{C}_{\mathrm{n}}$, then the total number of intersections in the graph is given by $\frac{1}{4} \sum_{\mathrm{i}=1}^{\mathrm{n}} \mathrm{x}_{\mathrm{i}}$.

THEOREM 2: A necessary condition on the minimum number of intersections $\mathrm{I}_{\mathrm{n}}$ of a complete graph $\mathrm{C}_{\mathrm{n}}$, is that it satisfy the relation

$$
\begin{array}{ll}
\mathrm{I}_{\mathrm{n}} \geqslant \frac{\mathrm{n}}{\mathrm{n}-4} \mathrm{I}_{\mathrm{n}-1} & n \geqslant 5 \\
I_{n}=0 & n \geqslant 4
\end{array}
$$

PRoof: The average number of intersections per vertex in $I_{n}$ is given by

$$
\frac{4 I_{n}}{n}
$$

The removal of a vertex with at least an average number of intersections must leave a $C_{n-1}$ whose intersection number is not less than $I_{n-1}$. Thus

$$
I_{n}-\frac{4 I_{n}}{n} \geqslant I_{n-1}
$$

from which the relation follows.

Repetition of the above relation gives:

$$
I_{n} \geqslant \frac{n(n-1)(n-2) \ldots(n-k+1)}{(n-4)(n-5)(n-6) \ldots(n-k-3)} I_{n-k}
$$

from which lower bounds on $I_{n}$ can be obtained if the minimal value of $I_{n-k}$ is known. Guy [2] writes the above relation as:

$$
I_{n} \geqslant I_{t}\left(\begin{array}{l}
n \\
t
\end{array}\right)\left(\begin{array}{l}
n-4 \\
t-4
\end{array}\right), 4<t \leqslant n
$$

An inductive argument may be used to show that the conjectured formula for $I_{n}$ holds for even $n$ given that it holds for the immediately preceeding odd case. This follows from

$$
\begin{gathered}
I_{k}-\frac{4}{k} I_{k} \geqslant I_{k-1} \\
I_{k} \geqslant \frac{k}{k-4} I_{k-1}=\frac{k}{k-4} \frac{(k-2)^{2}(k-4)^{2}}{64}=\frac{k(k-2)^{2}(k-4)}{64} .
\end{gathered}
$$

However, if $k$ is odd we have

$$
\begin{array}{r}
I_{k} \geqslant \frac{k}{k-4}\left[\frac{(k-1)(k-3)^{2}(k-5)}{64}\right]=\frac{(k-1)(k-3)^{2}}{64}\left[\frac{k(k-5)}{k-4}\right] \\
=\frac{(k-1)(k-3)^{2}}{64}\left[(k-1)-\frac{4}{k-4}\right]=\frac{(k-1)^{2}(k-3)^{2}}{64}-4 \frac{k-1}{k-4} \frac{(k-3)^{2}}{64}
\end{array}
$$

and induction does not work from the even to the odd case. 
THEOREM 3: $\mathrm{I}_{5}=1$.

Proof: (Kuratowski)[7].

THEOREM 4: $\mathrm{I}_{6}=3$.

Proof: Theorems 2 and 3 give $I_{6} \geqslant 3$. However $I_{6}=3$ is realizable.

THEOREM 5: Every complete graph on six vertices with a minimum number of intersections where its intersections are regarded as vertices, is isomorphic to the graph of the following figure:

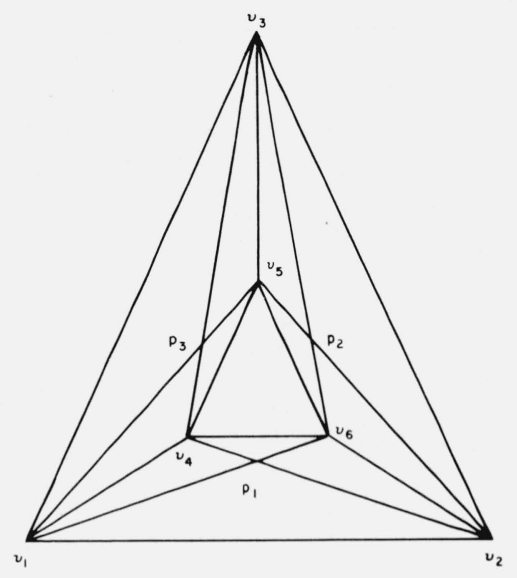

FigURE 1

Proof: Reference [9].

THEOREM 6: $\mathrm{I}_{7}=9$, with unique representation for the corresponding $\mathrm{C}_{7}$ to within stereographic projection.

Proof: Theorem 2 may be applied repeatedly to $I_{7}=3,4,5$, 6 each time obtaining a vertex with such a number of intersections defined by its star, that its removal together with its star leaves a $C_{6}$ behind with less than 3 intersections, contradicting Theorem 4.

We now prove $I_{7} \neq 8$ (the case $I_{7} \neq 7$ is analogous). By Theorem 1 we have $\frac{8 \times 4}{7}>4$. Thus there is at least one vertex with five intersections. Note that a vertex with 6 or more intersections would contradict Theorem 4 . The only possibility here is to take for example $x_{i}=5, i=1, \ldots$., 4 and $x_{i}=4, i=5, \ldots, 7$. In any case the removal of a vertex which defines 5 intersections leaves a subgraph $C_{6}$ which is minimal. Using the figure of Theorem 5 , we find that the 7 th vertex may fall in any one of three different regions and connected to the rest of the graph. The other regions are symmetric with these. The three regions are: inside the interior triangle $v_{4} v_{5} v_{6}$ (outside the exterior triangle $v_{1} v_{2} v_{3}$ is symmetric with this region by stereographic projection); in a triangle such as $v_{2} v_{6} p_{2}$; and finally in a triangle such as $v_{5} x_{6} p_{2}$. In all three cases the edges connecting the seventh vertex to any of the remaining vertices must introduce at least six additional intersections, contradicting the fact that the vertex was assumed to introduce exactly five intersections. Thus $I_{7} \geqslant 9$. However $I_{7}=9$ is realizable. Because $C_{7}$ with $I_{7}=9$ contains a $C_{6}$ with $I_{6}=3$ and this has a unique representation, our construction of $C_{7}$ is unique using symmetry arguments. See figure 5 .

THEOREM 7: $\mathrm{I}_{8}=18$.

Proof: From Theorems 2 and 6 we have $I_{8} \geqslant 18$. However $I_{8}=18$ is realizable. There is more than one representation.

THEOREM 8: $\mathrm{I}_{9}=36$.

Proof: By Theorem 2, $I_{9} \geqslant 33$. Let $I_{9}=x$. Since $\frac{18 \times 4}{8}=\frac{72}{8}=9$ and $\frac{19 \times 4}{8}=\frac{76}{8}>9$ we have for all three cases $x=33,34$, and 35 that the corresponding $C_{9}$ contains a minimal intersection $C_{7}$. 
Because a minimal intersection $C_{7}$ contains a minimal intersection $C_{5}$ and a minimal intersection $C_{3}$, we have a contradiction to theorem 2 reference [10]. Since $x=36$ is realizable, $I_{9}=36$. There is more than one representation.

THEOREM 9: $\mathrm{I}_{10}=60$.

Proof: By Theorems 2 and $8, I_{10} \geqslant 60$. However $I_{10}=60$ is realizable. There is more than one representation.

\section{Algebraic Formulation}

The intersection problem for $C_{n}$ may be algebraically formulated as follows:

Find nonegative integers $x_{i}, i=1, \ldots, n$ such that (1) There is a geometric realization (drawing in the plane) of $C_{n}$ with $x_{i}$ intersections associated with the $i$ th vertex and (2) these $x_{i}$ yield a minimum integer value to

$$
\frac{1}{4} \sum_{i=1}^{n} x_{i}
$$

such that this realizable minimum $I_{n}$ satisfies the condition

given in Theorem 2.

$$
I_{n} \geqslant \frac{n}{(n-4)} I_{n-1}
$$

We now prove that none of the components of the minimum solution vector $x=\left(x_{1}, \ldots, x_{n}\right)$ is zero $(n \geqslant 6)$. This is a corollary to:

THEOREM 10: The maximum number of intersections of $\mathrm{C}_{\mathrm{n}}$ is obtained by taking all its vertices on a polygon and joining them with edges in the interior of the polygon.

PRoof: Since each intersection is determined by four vertices, $\left(\begin{array}{l}n \\ 4\end{array}\right)$ is the maximum and the edges interior to the polygon give exactly this number of intersections since the diagonals of every quadrilateral intersect in the interior.

CoRollary: In a $\mathrm{C}_{\mathrm{n}} \mathrm{n} \geqslant 6$ with a minimum number of intersections, the edges connecting any vertex to the remaining $\mathrm{n}-1$ vertices must generate at least one intersection.

Proof: Otherwise all the vertices must be on a polygon and all their connecting lines must all be either inside or outside the polygon. However this gives a graph on $(n-1)$ vertices with a maximum number of intersections i.e., with $\left(\begin{array}{c}n-1 \\ 4\end{array}\right)$ intersections. But

$$
\left(\begin{array}{c}
n-1 \\
4
\end{array}\right)>\left\{\begin{array}{ll}
n(n-2)^{2}(n-4) / 64 & n \text { even } \\
(n-1)^{2}(n-3)^{2} / 64 & n \text { odd }
\end{array} \geqslant I_{n}\right.
$$

Thus at least one of the $n-1$ vertices must lie in a region defined by lines from the remaining $n-2$ vertices. In that case an edge from the $n$th vertex to the isolated vertex defines an intersection. This completes the proof.

\section{General Considerations}

Without the integer requirement on the value of $\frac{1}{4} \sum_{i=1}^{n} x_{i}$ or on its realizability, subject to the constraints we have:

THEOREM 11: A necessary condition for a minimum (maximum) of $\frac{1}{4} \sum_{\mathrm{i}=1}^{\mathrm{n}} \mathrm{x}_{\mathrm{i}}$ is that $\mathrm{x}_{\mathrm{i}}=\mathrm{x}_{\mathrm{j}}$ (or are as equal as possible in the sense of integer values) for all $\mathrm{i}$ and $\mathrm{j}$. 
Proof: Both minimum and maximum values are bounded above and below by positive quantities and hence $x=\left(x_{1}, \ldots, x_{n}\right)$ ranges over the lattice points of a bounded domain of $E^{n}$. The function $\prod_{i=1}^{n} x_{i}$ has a minimum over this domain. If we denote this minimum value by $G$ we have

$$
\frac{n}{4} G^{1 / n}=\frac{n}{4}\left(\prod_{i=1}^{n} x_{i}\right)^{1 / n} \leqslant \frac{n}{4} \sum_{i=1}^{n} \frac{x i}{n}
$$

The right side which gives the intersection number attains its minimum when the equality holds. Equality holds if and only if all the $x_{i}$ are equal. (See ref. [5] page 27.)

In the following relation:

$$
\frac{n}{4} \frac{\sum x_{i}}{n} \leqslant n^{2} / 4 \sum \frac{1}{x_{i}}
$$

the right side attains a maximum, $H$, as $x$ ranges over the lattice points. This gives

$$
\frac{n}{4} \sum \frac{x_{i}}{n} \leqslant n^{2} / 4 \sum \frac{1}{x_{i}}=H \text {. }
$$

The left attains its maximum when equality holds i.e., if and only if $x_{i}=x_{j}$ for all $i$ and $j$ (loc. cit. p. 26).

When $x_{i}=x_{j}=x$ for all $i$ and $j$ two difficulties may occur. The first is that

$$
\frac{1}{4} \sum_{i=1}^{n} x_{i}=\frac{n x}{4}
$$

may not be an integer. If it is an integer, it may not be possible to realize a complete graph such that all its vertices have the same number of intersections.

REMARK: Since the same basic formula $\frac{1}{4} \sum_{i=1}^{n} x_{i}$ holds for the number of intersections, this technique of equalizing the intersections among the vertices to obtain the minimum number would apply to any surface of genus $p$.

THEOREM 12: A complete graph with a maximum number of intersections has a realization with $\mathrm{x}_{\mathrm{i}}=\mathrm{x}_{\mathrm{j}}$ for all $\mathrm{i}$ and $\mathrm{j}$.

Proof: We have from the polygonal representation that

$$
x_{i}=\frac{1}{4}\left(\begin{array}{c}
n \\
4
\end{array}\right) i=1, \ldots, n
$$

We also have as a consequence of the results of the early part of the paper:

THEOREM 13: $\mathrm{C}_{\mathrm{n}}$ for $0 \leqslant \mathrm{n} \leqq 10, \mathrm{n} \neq 5,7$ with a minimum number of intersections has a realization with $\mathrm{x}_{\mathrm{i}}=\mathrm{x}_{\mathrm{j}}$ for all $\mathrm{i}$ and $\mathrm{j}$. For $\mathrm{n}=5,7$, all but one vertex have the same number of intersections. The odd vertex has a number of intersections which differs by unity from those of the other vertices.

Proof: We only need to prove the assertion for the cases $n=5$ and 7 . The other cases follow from the construction described in Theorem 1 reference [10]. The assertion for $n=9$ can be seen to hold in the following figure in which each $x_{i}=16$. The case $n=5$ follows from $I_{5}=1$ and the fact that an intersection is determined by 4 vertices. The odd vertex has no intersections. For $n=7$, since $I_{7}=9$ and 7 does not divide 36 not all $x_{i}$ are equal. Thus $x_{i}=5 i=1, \ldots, 6$ and $x_{7}=6$ see figure.

REMARK: In reference [1] a realization scheme which utilizes a Hamilton Circuit through the vertices with no intersections on its edges is described. This differs from the two concentric polygons scheme illustrated below (see figs. 6 and 8) and completely justified in reference [10]. 
We also have:

THEOREM 14: $\mathrm{C}_{\mathrm{n}}$ for $\mathrm{n}$ even, whose intersection number is given by the conjecture and whose representation is given by the construction procedure of Theorem 1 reference [10] has a realization with $\mathrm{x}_{\mathrm{i}}=\mathrm{x}_{\mathrm{j}}$ for all $\mathrm{i}$ and $\mathrm{j}$.

Proof: It follows from Theorem 1 reference [10] that $x_{i}=x_{j}$ for all $i$ and $j$.

\section{Combinatorial Symmetry}

This section is intended to give general combinatorial form to the conjecture at the beginning of the next section and, to some extent, to provide motivation for that conjecture. The reader may, however, omit this section and proceed directly to section 5 .

In geometry, symmetry is defined by means of isometries called symmetry operations (and their groups of automorphisms) which leave a figure unchanged or invariant while permuting its parts $[1 \mathrm{a}, 111]$. The dimensions, translations and rotations, of the figure and its parts play an important role in characterizing its symmetry.

Here we introduce symmetry in a slightly more general sense. This symmetry depends on rules of construction applied to elements of a set appropriately partitioned into disjoint subsets together with a semigroup of maps between the members of the partition and lead to a particular combinatorial property that is preserved under the semigroup. Thus the symmetry operations leave the combinatorial property unchanged while the members of the partition of the set are interchanged elementwise.

An example is given by the representation of the maximum number of intersections of $C_{n}$. Here the set is partitioned into its singletons (the trivial partition), each member being a vertex. The representation is invariant to a transformation which carries a vertex and its star to another vertex and its star maintaining the order of the edges as they appear in the star. In general we can consider any partition.

We note in the following representation (fig. 2) of $C_{k} k=4,5,6, \ldots$. with a maximum number of intersections that the removal of any vertex and its star from $C_{n}$ leaves a $C_{n-1}$ with a maximum number of intersections. Here each $C_{n}$ contains all lower order $C_{k}$ 's $k=1,2, \ldots, n$, each of which occurs $\left(\begin{array}{l}n \\ k\end{array}\right)$ times.
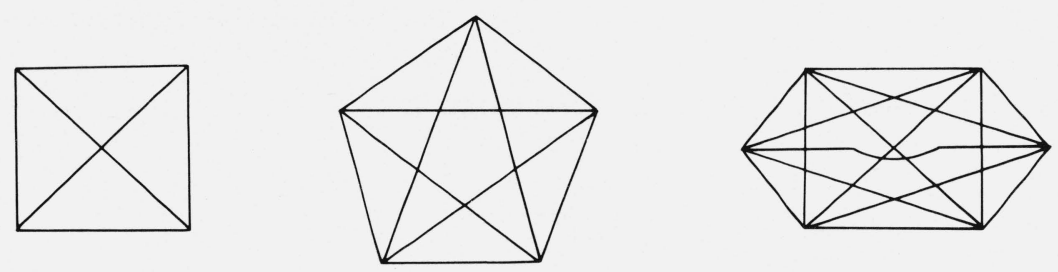

FIGURE 2

\section{A Regeneration Principle of Combinatorial Symmetry}

We find the following observation useful. It is that maximality is preserved when additional vertices are inserted in the figure having smaller number of vertices. Thus when symmetry is retained in the construction of a figure which is an extension of a combinatorially symmetric figure on a smaller number of elements and having a special character of the combinatorial property (e.g., maximality of intersections) then that special property is also preserved for the larger set. The process of extension may also be regarded as a superposition of a figure for a larger set of elements over the corresponding figure for a smaller set. In terms of the partition and the semigroup of maps, the insertion of vertices is an extension of the original figure, its partition and the semigroup of maps. 
Thus the larger figure is an extension of a smaller figure with preservation of symmetry. The smaller one can then be obtained from the larger one by the removal of appropriate elements and yet smaller figures may be derived from the latter and so on. In this manner we obtain a descending sequence of figures each of which retains the relative combinatorial property. We refer to the process of extension with symmetry and preservation of relative properties as regeneration.

The possibility of symmetric regeneration suggests:

Conjecture (Regeneration Principle): If a given combinatorial property is known to hold for the smallest order symmetric construction applied to a partition of a set and also hold for an appropriate subset of the family of its immediate ascendants (to insure that the rules of symmetric construction permit continued ascendance with preservation of the property) then the property holds for an arbitrary ascendant.

Possibly the most essential criterion for developing symmetrization rules for minimal or maximal $C_{n}$ is based on the following:

DEFINITION: A symmetric realization of a complete graph is a symmetric drawing in the plane for which $\sum_{\mathbf{i}, \mathrm{j}=1}^{\mathrm{n}}\left(\mathrm{x}_{\mathrm{i}}-\mathrm{x}_{\mathrm{j}}\right)^{2}=\min$.

Returning to the problem of minimal $C_{n}$, suppose that $n$ is even. Symmetrization from $C_{n-2}$ to $C_{n}$ by introducing a pair of vertices must conform to the symmetry criteria already used to construct a symmetric $C_{n-2}$. Geometrically $C_{n}$ is said to have the symmetric properties of $C_{n-2}$ if its vertices can be divided into disjoint pairs with preservation of mutual relationship between pairs.

The pairs are interchangeable according to (1) the number of intersections sustained on their edges; (2) the rule or scheme according to which these edges are drawn. Thus a pair with its connecting edges (a double star) to the remaining $C_{n-2}$ forms a configuration which is identical with that of any of the other $\frac{n}{2}-1$ pairs. An edge in the double star of a pair has the same number of intersections following the rules of the symmetric construction as the corresponding edge in the double star of any other pair.

The removal of one vertex of any pair together with its star from $C_{n}, n$ even, must yield the desired $C_{n-1}$ with the conjectured number of intersections. To obtain $C_{n-2}$ the other vertex of the pair must be removed.

The construction of a symmetric $C_{n}$ for the conjectured value of $I_{n}$ and satisfying the pairing requirement has been described in detail in the proof of Theorem 1 of reference [10]. The pairs are identified as in figure 8 . One vertex belongs to the outer polygon and its companion is the farthest vertex (or one of the farthest if there is a tie) on the inner polygon.

The removal of any one of the pairs of $C_{n}$ naturally leaves a symmetric $C_{n-2}$. The addition of pairs to a lower order $C_{n}$ must follow the rules of symmetry used to construct that $C_{n}$ from its antecedent. If the Regeneration Principle holds then the graph is minimal. It can be shown to satisfy the conjectured value of $I_{n}$ in 4 ways. (1) Two ways are given each by one of the two theorems of reference [10]; (2) since the pairs are chosen in the designated manner, it is trivial to note that all their corresponding $C_{4}$ 's are minimal and hence the discussion on pages 574-575 of reference [10] applies; (3) see section 5 .

This conjecture supports a counting method used below. A $C_{8}$ is the smallest complete subgraph of a $C_{n}$ in which all three types of intersections which can arise between pairs occur. These are intersections (a) between two pairs, (b) between three pairs i.e., the intersecting edges have their end points among the three pairs and (c) between four pairs (each of the four end points belongs to a different pair). 


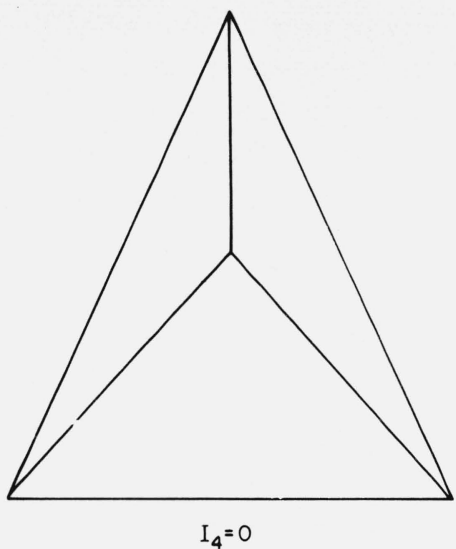

Fiǵure 3

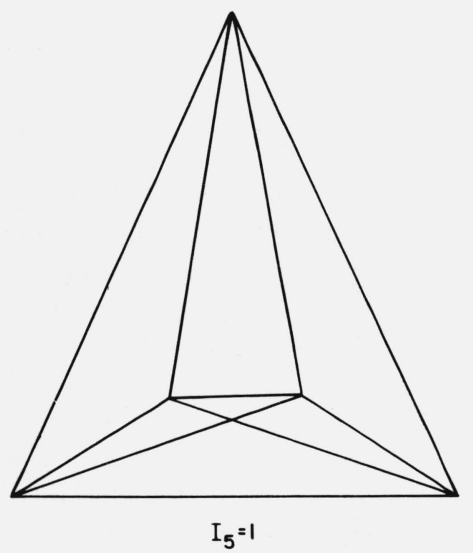

Figure 4

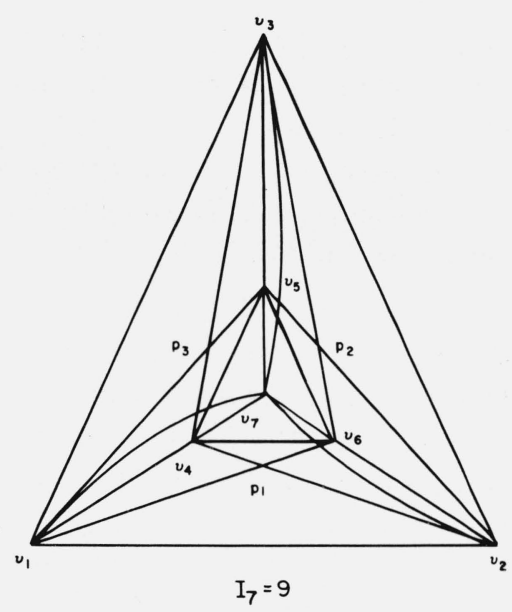

Figure 5

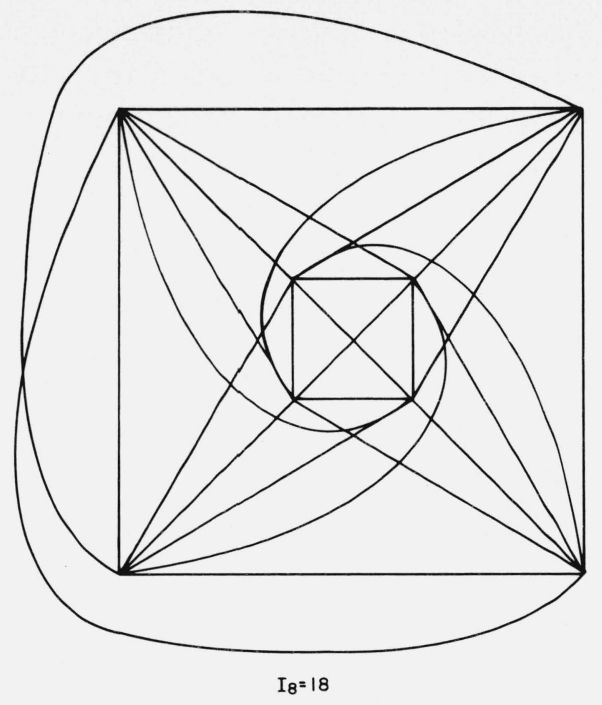

Figure 6

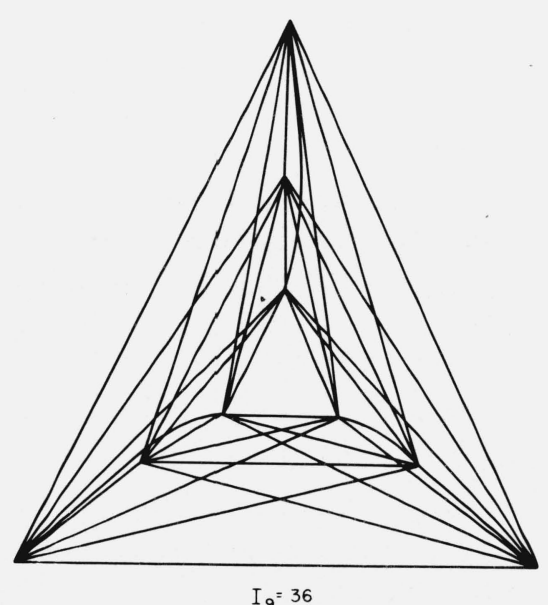

Figure 7



$I_{10}=60$

Figure 8 


\section{Conjecture}

If $\mathrm{C}_{\mathrm{n}}$ has a minimal intersection representation from which one may choose $\mathrm{r}=\left[\frac{\mathrm{n}}{2}\right]$ pairs $P_{1}, \ldots, P_{\mathrm{r}}$ of vertices of $\mathrm{C}_{\mathrm{n}}$ such that: If $\mathrm{C}_{2 \mathrm{~s}}$ is the full subgraph of $\mathrm{C}_{\mathrm{n}}$ determined by $P_{1} \cup$. . $\cup P_{\mathrm{s}}(1 \leqslant \mathrm{~s} \leqslant \mathrm{r}-1)$, then $\mathrm{C}_{2 \mathrm{~s}}$ is minimal. Moreover, if $\mathrm{n}$ is odd with vertices $v \cup P_{1} \cup \ldots \cup P_{\mathrm{r}}$ then any $\mathrm{C}_{7}$ determined by vertices $v \cup P_{i} \cup P_{\mathrm{j}} \cup P_{\mathrm{k}}, \mathrm{i} \neq \mathrm{j} \neq \mathrm{k}$, is minimal.

Remark: Note that the Regeneration Principle implies the Conjecture.

THEOREM 15: If the above Conjecture is true (and hence if the Regeneration Principle is true), then $\mathrm{I}_{\mathrm{n}}$ has the value conjectured.

Proof By INDUCTION ON $n$ : We assume that $I_{m}$ has the conjectured value for $m<n+2$ and assert that $I_{n+2}$ does also. If $n$ is even then $n+1$ is odd so $I_{n+1}$ has the conjectured value and by the remark after theorem $2, I_{n+2}$ has the conjectured value. Therefore we suppose that $n$ is odd say $n=2 k+1$.

Let $C_{n+2}$ be arbitrary and use the conjecture to choose pairs $P_{1}, \ldots, P_{k+1}$ of vertices with one vertex $v$ left over. By induction $C_{n+1}=C_{n+2}-v$ has at least $I_{n+1}$ intersections. Since $I_{n+2}-I_{n+1}$ $=k\left(\begin{array}{c}k+1 \\ 2\end{array}\right)$, we must show that adding the vertex $v$ to this $C_{n+1}$ introduces at least $k\left(\begin{array}{c}k+1 \\ 2\end{array}\right)$ new intersections. Now by the conjecture the $C_{n-1}$ determined by $P_{1} \cup \ldots \cup P_{k}$ is minimal so the addition of $v$ to this $C_{n-1}$ must introduce at least $(k-1)\left(\begin{array}{l}k \\ 2\end{array}\right)$ intersections. Thus, we need only show that

$$
k\left(\begin{array}{c}
k+1 \\
2
\end{array}\right)-(k-1)\left(\begin{array}{l}
k \\
2
\end{array}\right)=3\left(\begin{array}{l}
k \\
2
\end{array}\right)+k
$$

new intersections are introduced by the addition of $v$ to the $C_{n+1}$ determined by $P_{1} \cup \ldots \cup P_{k+1}$ but not merely by the addition of $v$ to the $C_{n-1}$ determined by $P_{1} \cup \ldots \cup P_{k}$.

To see that we can do this, consider any pair of pairs $P_{i}, P_{j}, 1 \leqslant i \neq j \leqslant k$. By the conjecture $v \cup P_{i} \cup P_{j} \cup P_{k+1}$ determine a minimal $C_{7}$ with nine intersections. It follows from theorem 6 that exactly three of these intersections involve $v$ and all three of the pairs $P_{i}, P_{j}, P_{k+1}$. Thus we obtain $3\left(\begin{array}{l}k \\ 2\end{array}\right)$ new intersections as $\{i, j\}$ ranges over $\{1, \ldots, k\}$. We obtain at least $k$ more intersections by taking all $C_{5}$ s of the form $P_{i} \mathrm{P}_{k+1} v$. This completes the proof.

\section{Generalization}

The minimum intersection problem for $\mathrm{C}_{\mathrm{n}}$ is a special case of the following general problem. Consider a connected graph $\mathrm{G}$ with $\mathrm{n}$ vertices joined by edges in the plane as follows: each vertex is joined to $\mathrm{k}$ other vertices $(2 \leqslant \mathrm{k} \leqslant \mathrm{n}-1)$ in such a way that a maximum number of vertices has degree $\mathrm{k}$. If any two edges can cross at most once at a point other than a vertex, determine the minimum number of intersections $\mathrm{I}_{\mathrm{n}}(\mathrm{k})$ of the edges of $\mathrm{G}$.

The solution of this problem should follow from our previous discussion of symmetric realization. Our existing realization may be used to remove the necessary number of edges.

It is clear for example that the removal of the edges joining the prescribed pairs in the even case deletes the largest number of intersections. In the odd case one vertex must have no edge removed otherwise another vertex would have $(n-2)$ edges removed. The operation of removing an edge per vertex is continued. This gives

$$
\begin{aligned}
I_{n}(n-2)= & n \text { even } \\
& \frac{n(n-2)(n-4)(n-6)}{64} \\
& n \text { odd }
\end{aligned}
$$




$$
I_{n}(n-3)=\begin{array}{cc}
\frac{n(n-4)(n-6)^{2}}{64} & n \text { even } \\
\frac{(n-3)^{2}(n-5)^{2}}{64} & \\
\cdot \cdot \cdot & \\
I_{n}(3)=0 & \\
I_{n}(2)=0 \\
I_{n}(1)=0 \\
I_{n}(0)=0 .
\end{array}
$$

Induction on $k$ may be useful to go from the lower cases to higher ones.

REMARK: If the regeneration principle holds, then the proof of Zarankiewicz's problem for the minimum intersection number of edges joining every vertex of one set to every vertex of another set in a bipartite graph would follow from his symmetric construction.

I am indebted to I. Heller, and Paul Kainen for helpful comments and suggestions.

\section{References}

[1] Blažek, J., and Koman, M., A minimal problem concerning complete plane graphs, in Theory of Graphs and Its Applications, Proc. Symp. Smolenice, June 1963 (Academic Press, New York, 1964).

[la] Coxeter, H.S.M., Introduction to Geometry, (John Wiley \& Sons, Inc., New York, N.Y., 1962).

[2] Guy, R. K., A combinatorial problem, Bull. Malayan Math. Soc. 7, No. 2, 68-72 (July 1960).

[3] Guy, Richard K., Jenkyns, Tom, and Schaer, Johathan, The toroidal crossing number of the complete graph, University of Calgary, Department of Mathematics, Research Paper No. 18, May 1967.

[4] Harary, F., and Hill, A., On the number of crossings in a complete graph, Proc. Edinburgh Math. Soc. 13, $333-338$.

[5] Hardy, G. H., Littlewood, J. E., and Polya, G., Inequalities (Cambridge University Press, 1934).

[6] Kainen, Paul, On a problem of Erdïs, J. Comb. theory 5, No. 4, 374-377 (Dec. 1968)

[7] Kuratowski, Kasimir, Sur le probleme des courbes gauches en topologie, Fund. Math., 15, 271-283 (1930).

[8] Moon, J. W., On the distribution of crossings in random complete graphs, J. Soc. Indust. Appl. Math. 13,506-510 (1965).

[9] Saaty, T. L., Remarks on the four color problem; the Kempe catastrophe, Math. Mag. (Jan. 1967).

[10] Saaty, T. L., Two theorems on the minimum number of intersections for complete graphs, Journal of Combinatorial Theory, 2, No. 4, 571-584 (June 1967).

[11] Weyl, Hermann, Symmetry (Princeton University Press, 1952).

[12] Zarankiewicz, K., On a problem of P. Turan concerning graphs, Fund. Math. 41, 1954, 137-145.

(Paper 73B2-298) 


\section{Publications of the National Bureau of Standards*}

\section{J. Res. NBS 73 A (Phys. and Chem.), No. 2 (Mar.-Apr. 1969), $\$ 1.00$}

Color representation of electron microprobe area-scan images by a color separation process. H. Yakowitz and K. F. J. Heinrich. Configurations $3 d^{n} 4 p$ in singly ionized atoms of the iron group. C. Roth.

Configurations $3 d^{n} 4 p+3 d^{n-1} 4 s 4 p$ in Sc II, Ti II, and V II. C. Roth.

Effective interactions in the even configurations of the third spectra of the iron group. Y. Shadmi, E. Caspi, and J. Oreg.

Test of a kinetics scheme: Emission in $\mathrm{H}\left({ }^{2} \mathrm{~S}\right)+\mathrm{NO}\left({ }^{-1}\right)$. M. Krauss.

A nuclear magnetic resonance and relaxation study of dimethoxyborane. T. C. Farrar and T. Tsang.

The effects of low energy irradiation on organometallics. Organometal halides of group IV A. F. E. Brinckman, G. F. Kokoszka, and N. K. Adams, Jr.

Measurements of gaseous diffusion coefficients by a gas chromatographic technique. S. P. Wasik and K. E. McCulloh.

Phase relations in the Ru-Ir-O $\mathrm{O}_{0}$ system in air. C. L. McDaniel and S. J. Schneider.

Heats of reaction of natural rubber with sulfur. N. Bekkedahl and J. J. Weeks.

A table of rotational constants of symmetric top molecules giving rise to microwave spectra. M. S. Lojko and Y. Beers.

Energy levels, wave functions, dipole and quadrupole transitions of $\mathrm{Fe}^{+++}$ions in sapphire. J. Lewiner and P. H. E. Meijer.

Bullis, W. M., Measurement of carrier lifetime in semiconductors, An annotated bibliography covering the period 1949-1967, Tech. Note 465 (Nov. 1968), 60 cents.

Cassidy, E. C., Abramowitz, S., Beckett, C. W., Investigations of exploding wire process as a source for high temperature studies, Mono. 109 (Nov. 1968), 55 cents.

Cord, M. S., Petersen, J. D., Lojko, M. S., Haas, R. H., Microwave spectral tables, Polyatomic molecules without internal rotation, Mono. 70, Vol. IV (Oct. 1968), \$5.50.

Flynn, D. R., Peavy, B. A., Jr., Ed., Thermal Conductivity, Proceedings of Seventh Conference. National Bureau of Standards, Gaithersburg, Md., Nov. 13-16, 1967, Spec. Publ. 302 (Sept. 1968), $\$ 6.25$.

Janz, G. J., Dampier, F. W., Lakshminarayanan, G. R., Lorenz, P., K., Tomkins, R. P. T., Molten salts: Volume 1, Electrical conductance, density, and viscosity data, NSRDS-NBS15 (Oct. 1968), $\$ 3.00$.

Kropschot, R. H., Birmingham, B. W., Mann, D. B., Ed., Technology of liquid helium, Mono. 111 (Oct. 1968), \$2.00.

Lederer, P. S., NBS interagency transducer project, Tech. Note 469 (Oct. 1968), 30 cents.

Lutz, G. J., Boreni, R. J., Maddock, R. S., Meinke, W. W., Ed., Activation analysis: A bibliography, Tech. Note 467, Parts 1 and 2 (Sept. 1968), \$6.50 set.

McClenon, R. C., Hilsenrath, J., Reform: A general-purpose program for manipulating formatted data files, Tech. Note 444 (Aug. 1968), 40 cents.

Moiseiwitsch, B. L., Smith, S. J., Electron impact excitation of atoms, NSRDS-NBS 25 (Aug. 1968), $\$ 2.00$.

Schaffer, R., Ed., Organic Chemistry Section: Summary of activities July 1967 to June 1968, Tech. Note 457 (Sept. 1968), \$1.25.

Stevens, M. E., Nonnumeric data processing in Europe, A field trip report, August-October 1966, Tech. Note 462 (Nov. 1968), 65 cents.

Taylor, J. K., Ed., Microchemical Analysis Section: Summary of activities July 1967 to June 1968, Tech. Note 455 (Oct. 1968), $\$ 1.25$.
Technical highlights of the National Bureau of Standards, Annual Report, Fiscal Year 1968, Spec. Publ. 308 (Nov. 1968), \$1.00.

Altschuler, H. M., Comments on "An improved method for meas. uring scattering parameters of nonreciprocal two-ports," IEEE 'Trans. Microwave Theory Tech. M'TT-16, No. 4, 261-262 (Apr. 1968).

Arenhovel, H., General formulas for describing the absorption of polarized photons by oriented nuclei, Phys. Rev. 171, No. 4, 1212-1216 (July 20, 1968).

Bach, R. L., Caswell, R. S., Energy transfer to matter by neutrons, Radiation Res. 35, No. 1, 1-25 (July 1968).

Bennett, L. H., Mebs, R. W., Watson, R. E., Solute Knight shifts in noble metals, Phys. Rev. 171 , No. 3, 611-626 (July 15, 1968).

Brauer, G. M., McLaughlin, R., Huget, E. F., Aluminum oxide as a reinforcing agent for zinc oxide-eugenol-o-ethoxybenzoic acid cements, J. Dental Res. 47, No. 4, 622-628 (July-Aug. 1968).

Birmingham, B. W., Ed., A Report on the 1967 Applied Superconductivity Conference, Cryogenics 8, No. 3, 176-179 (June 1968).

Bowen, R. L., Paffenbarger, G. C., Mullineaux, A. L., A laboratory and clinical comparison of silicate cements and a direct-filling resin: A progress report, J. Prosthetic Dentistry 20, No. 5, 426437 (Nov. 1968).

Branscomb, L. M., Physics and the nation in a crystal ball, Phys. Today 21, No. 8, 23-28 (Aug. 1968).

' Brooks, R., Horton, A. T., Torgesen, J. L., Occlusion of mother liquor in solution-growth crystals, J. Crystal Growth 2, 279-283 (1968).

Bullis, W. M., Measurement problems in microcircuit processing, (Proc. Government Microcircuit Applications Conf., Gaithersburg, Md., Oct. 1-3, 1968) GOMAC Digest 1, 215-217 (Office of Naval Research, W ashington, D.C., Oct. 1968).

Cassidy, E. C., Abramowitz, S., Time-resolved emission and absorption studies of exploding wire spectra (Proc. 4th Conf. Exploding Wires, Boston, Mass., Oct. 18-21, 1967), Book, Exploding Wires, 4, $109-124$ (Plenum Press Inc., New York, N.Y., 1968).

Christ, B. W., Giles, P. M., On the detection of retained austenite in high-carbon steels by $\mathrm{Fe}^{57}$ Mössbauer spectroscopy, with appendix, Trans. Met. Soc. AIME 242, 1915-1925 (Sept. 1968).

Christ, B. W., Smith, G. V., Effects of impurities on yield and flow strength of zone refined iron, Mem. Sci. Rev. Met. LXV, 208-223 (June 15, 1968).

Clark, A. F., Powell, R. L., Longitudinal magnetoresistance of pure single-crystal copper, Phys. Rev. Letters 21, No. 12, 802-804 (Sept. 16, 1968).

Corruccini, R. J., Principles of thermometry (measurement of temperature), Book, Treatise on Analytical Chemistry, Ed. I. M. Kolthoff, P. J. Elving, and E. B. Sandell, Part I, Theory and Practice, 8, Chapter 87, 4937-4990 (Interscience Publishers, New York, N.Y., 1968).

Danielson, B. L., An optical Faraday rotation technique for the determination of magnetic relaxation times, IEEE Trans. Mag. MAG-4, No. 2, 176-178 (June 1968).

Dibeler, V. H., Liston, S. K., Mass-spectrometric study of photoionization. IX. Hydrogen cyanide and acetonitrile, J. Chem. Phys 48, No. 10, 4765-4768 (May 15, 1968).

Dibeler, V. H., Liston, S. K., Mass spectrometric study of photoionization. XII. Born trifluoride and diboron tetrafluoride, Inorg. Chem. 7, No. 9, 1742-1746 (Sept. 1968).

Dick, C. E., Motz, J. W., Inelastic scattering cross sections for 200 and 400-keV electrons, Phys. Rev. 171, No. 1, 75-80 (July 5, 1968).

Dickson, R. W., Spinner, S., An improved method for the determination of torsional and flexural resonance frequencies of cylindrical specimens, J. Mater. 3, No. 3, 716-724 (1968).

Drechsel, D., On the model-independent evaluation of inelastic electron scattering data at low momentum transfer, Nucl. Phys. A1 13, No. 3, 665-675 (June 7, 1968). 
Edmiston, C., Krauss, M., Pseudonatural orbitals as a basis for the superposition of configurations. II. Energy surface for linear $\mathrm{H}_{3}$, J. Chem. Phys. 49, No. 1, 192-2u5 (July 1, 1968).

Fano, U., Cooper, J. W., Spectral distribution of atomic oscillator strengths, Rev. Mod. Phys. 40, No. 3, 441-507 (July 1968).

Fatiadi, A. J., Acylation of tetrahydroxy-p-benzoquinone, J. Chem. Eng. Data 13, No. 4, 591-593 (Oct. 1968).

Fatiadi, A. J., Bromine oxidation of inositols for preparation of inosose phenylhydrazones and phenylosazones, Carbohydrate Res. 8, 135-147 (1968).

Florin, R. E., Sicilio, F., Wall, L. A., The paramagnetic species from titanous salts and hydrogen peroxide, J. Phys. Chem. 72, No. 9 , 3154-3161 (Sept. 1968).

Folman, M., Klein, R., Second layer migration with trapping on first layer sites: hydrogen, nitrogen, and carbon monoxide on tungsten, Surface Sci. 11, 430-442 (1968).

Gebbie, K. B., Thomas, R. N., Non-LTE diagnostic stellar spectroscopy. I. Conceptual and analytical background, Astrophys. J. 154, 271-283 (Oct. 1968).

Gebbie, K. B., Thomas, R. N., Non-TLE diagnostic stellar spectroscopy. II. On the Schuster mechanism for the production of emission lines, Astrophys. J. 154, 285-296 (Oct. 1968).

Green, M. S., Generalized Ornstein-Zernike approach to critical phenomena, J. Math. Phys. 9, No. 6, 875-890 (June 1968).

Hardy, S. C., Coreill, S. R., Morphological stability of a growing cylindrical crystal of ice, J. Appl. Phys. 39, No. 7, 3505-3507 (June 1968).

Hiza, M. J., Heck, C. K., Kidnay, A. J., Liquid-vapor and solid-vapor equilibrium in the system hydrogen-ethylene, Proc. Symp. Cryogenic Advance in the Space Program, Tampa Florida, May 19-22, 1968, 64, No. 88, 57-65 (American Institute of Chemical Engineering, New York, N.Y., 1968).

Huget, E. F., Brauer, G. M., Reactivity of the components of tooth structure, J. Colloid Interface Sci. 27, No. 4, 714-721 (Aug. 1968).

Hummer, D. G., Rybicki, G. B., Redshifted line profiles from differentially expanding atmospheres, Astrophys. J. 153, L107L110 (Aug. 1968).

Hyde, K. E., Gordon, G., Kokoszka, G. F., Magnetic properties of some binuclear copper (II) con.plexes, J. Inorg. Nucl. Chem. 30, 2155-2161 (1968)

Iverson, W. P., Microbiological corrosion, Proc. Conf. Corrosion and Protection of Pipes and Pipelines, London, England, June 13, 1968 (Technical Exhibited Ltd., London, England, 1968).

Jacox, M. E., Milligan, D. E., Matrix-isolation study of the infrared spectrum of the free radical $\mathrm{F}_{2} \mathrm{CN}$, J. Chem. Phys. 48, No. 9, 4040-4046 (May 1, 1968).

Jespersen, J. L., Kamas, G., Gatterer, L. E., MacDoran, P. F., Satellite VHF transponder time synchronization, Proc. IEEE 56, No. 7, 1202-1206 (July 1968).

Johannesen, R. B., Gordon, C. L., Titanium (IV) bromide (titanium tetrabromide), Book, Inorganic Syntheses IX, Chapt. IV, 46-50 (McGraw-Hill Book Co., New York, N.Y., 1967).

Joiner, B. L., Rosenblatt, J. R.. The mean deviation and range for $n=3$, Am. Stat. 22, No. 4, 37-38 (Oct. 1968).

Judd, D. B., Color science and the paint industry, J. Paint Technol. 40, No. 525, 470-477 (Oct. 1968).

Kirby, R. K., Rothrock, B. D., Thermal expansion of vitreous selenium from $-190^{\circ}$ to $+30^{\circ} \mathrm{C}$, J. Äm. Ceram. Soc. 5î , No. 9, 535 (Sept. 1968).

Kokoszka, G. F., Linzer, M., Gordon, G., Electron paramagnetic resonance spectra of polycrystalline dimeric complexes. Copper propionate monohydrate and zinc-doped copper propionate monohydrate, Inorg. Chem. 7, No. 9, 1730-1735 (1968).

Krause, R. F., Jr., Douglas, T. B., The heats of formation of $\mathrm{AlClF}_{2}$ and $\mathrm{AlCl}_{2} \mathrm{~F}$ from subliming $\mathrm{AlF}_{3}$ in the presence of $\mathrm{AlCl}_{3}$ vapor, $\mathrm{J}$. Phys. Chem. 72, No. 10, 3444-3451 (Oct. 1968).

$\mathrm{Ku}$, H. H., Expressions of imprecision, systematic error, and uncertainty associated with a reported value, Meas. Data 2, No. 4, 72-77 (July-Aug. 1968).

Kuriyama, M., Theory of x-ray diffraction by a distorted crystal. II. Scattering amplitude for a wave packet of finite size, J. Phys. Soc. Japan 25, No. 3, 846-856 (Sept. 1968).

Kushner, L. M., The National Bureau of Standards and the Fire Research and Safety Act of 1968, Fire J. 62, No. 5, 32 (Sept. 1968).

Kuyatt, C. E., Measurement of electron scattering from a static gas target, Book, Methods of Experimental Physics. Vol. 7. Atomic and Electron Physics, Chapt. 1. Electron-Atom Interactions, Ed. B.
Pederson and W. L. Fite, pp. 1-43 (Academic Press Inc., New York, N.Y., 1968).

McCaa, W. D., Jr., Nahman, N. S., Phenomena and devices, frequency and time-domain analysis of a superconductive coaxial line using the two-fluid model, J. Appl. Phys. 39, No. 6, 2592-2596 (May 1968).

McClintock, M., Jennings, D. A., Mizushima, M., Contribution to the Raman line profile in liquids from molecular reorientation, Phys. Rev. Letters 21, No. 5, 276-278 (July 29, 1968).

Macedo, P. B., Napolitano, A., Inadequacies of viscosity theories for $\mathrm{B}_{2} \mathrm{O}_{3}$, J. Chem. Phys. 49, No. 4, 1887-1895 (Aug. 15, 1968).

Madey, T. E., Yates, J. T., Jr., Desorptıon by electron impact: oxygen adsorbed on tungsten, Surface Sci. 11, 327-351 (1968).

Mazur, J., McCrackin, F. L., Monte Carlo studies of configurational and thermodynamic properties of self-interacting linear polymer chains, J. Chem. Phys. 49, No. 2, 648-665 (July 15, 1968).

Mears, T. W., Young, D. S., Measurement and Standard Reference Materials in clinical chemistry, Am. J. Clin. Pathol. 50, No. 4, 411-421 (Oct. 1968).

Milligan, D. E., Jacox, M. E., Matrix-isolation study of the reaction of $\mathrm{F}_{2}$ and of $\mathrm{F}$ atoms with NCN. The infrared spectra of the species $\mathrm{NF}_{2} \mathrm{CN}$ and FNCN, J. Chem. Phys. 48, No. 11, 4811-4816 (June 1,1968 ).

Moore, G. A., Application of computers to quantitative analysis of microstructures (Proc. Third Intern. Materials Symp., Berkeley, Calif., June 13, 1966), Book, Ceramic Microstructures, Ed. R. M. Fulrath and J. A. Pask, Chapt. 3, pp. 71-120 (John Wiley and Sons, Inc., New York, N.Y., 1968).

Moore, G. A., Automatic scanning and computer processes for the quantitative analysis of micrographs and equivalent subjects (Proc. Symp. on Automatic Photo Interpretation, Washington, D.C., June 1967), Book, Pictorial Pattern Recognition, pp. 275326 (Thompson Book Co., Washington, D.C., 1968).

Munis, R. H., Finkel, M. W., Goniometric measurements of infrared transmitting materials, Appl. Opt. 7, No. 10, 2001-2004 (Oct. 1968).

Omar, A., Peterson, R. L., On the validity of the Boltzmann relaxation equation, Physica 39, No. 1, 156-160 (July 1, 1968).

Pechukas, P., Generalized Langevin equation of Mori and Kubo, Phys. Rev. 164, No. 1, 164-175 (Dec. 5, 1967).

Pecker, J-C., Thomas, R. N., Saturation in Fraunhofer lines, Observatory Letter 88, No. 964, 115-117 (June 1968).

Peiser, H. S., The relative magnitude of jump rates for point-defect paths between equivalent sites around a trap, J. Korean Phys. Soc. 1, No. 1, 5-8 (Mar. 1968).

Peterson, R. L., Short-range magnetic order in a modified Weiss molecular-field theory, Phys. Rev. 171, No. 2, 586-590 (July 10, 1968).

Potzick, J., ac-dc Regulator-modulator, Rev. Sci. Instr. 39, No. 8, 1219-1220 (Aug. 1968).

Ray, C. D., Tsukishima, T., Hyland, R. N., McLane, C. K., Carpenter, R. J., Analysis of a frequency-swept one-bit digital correlator, J. Appl. Phys. 39, No. 8, 3534-3542 (July 1968).

Romanoff, M., Performance of ductile-iron pipe in soils, J. Am. Water Works Assoc. 60, No. 6, 645-655 (june 1968).

Santoro, A., Weir, C. E., Block, S., Piermarini, G. J., Absorption corrections in complex cases. Application to single crystal diffraction studies at high pressure, J. Appl. Cryst. 1, Part 2, 101-107 (June 1968).

Saunders, J. B., Bruening, R. J., A new interferometric test and its application to the 84-in. reflecting telescope at Kitt Peak National Observatory, Astron. J. 73, No. 6, 415-531 (Aug. 1968).

Scharf. K., Steady-state response of silicon radiation detectors to $\mathrm{X}$ and gamma rays, Proc. Symp. Physics and Nondestructive Testing, ITT Research Institute, Chicago, Ill., Sept. 27-30, 1966, 1, 235-298 (Air Force Materials Laboratory, Wright-Patterson Air Force Base, Ohio, 1968).

Schmidt, L. B., Case, W. E., Harrington, R. D., Surface dependence of ferrimagnetic resonance fields in polycrystalline $\mathrm{NiAl}$ ferrite, Proc. IEEE 56, No. 7, 1236-1237 (July 1968).

Shumaker, J. B., Jr., Popenoe, C. H., Experimental test of $\mathrm{H}_{\beta}$ Stark-broadening theory at high electron densities, Phys. Rev. Letters 21, No. 15, 1046-1048 (Oct. 7, 1968).

Sieck, L. W., Continuum emission from zenon in the vapor phase induced by absorption of $1470-\AA$ radiation, J. Phys. Chem. 72 , No. 9, 3129-3133 (Sept. 1968). 
Simmons, J. A., Cioriell, S. R., Solution of the Stetan problem for whisker evaporation, J. Appl. Phys. 39, No. 7, 3459-3463 (June 1968).

Steiner, B., The cross section for the photodetachment of electrons from I-, Phys. Rev. 173, No. 1, 136-142 (Sept. 5, 1968).

Stevens, M. E., Progress and prospects in mechanized indexing, (Proc. Symp. on Meçhanized Abstracting and Indexing, Moscow, U.S.S.R., Sept. 28-Oct. 1, 1966), UNESCO/NS/209 (United Nations, Educational Scientific and Cultural Organization, Paris, France, Apr. 1967).

Steward, W. G., Smith, R. V., Brennan, J. A., Cooldown time for simple cryogenic pipelines, (Proc. Tenth Midwestern Mechanics Conf., Colorado State University, Fort Collins, Colo., Aug. 21-23, 1967), Book, Developments in Mechanics 4, 1513-1525 (Johnson Publ. Co., Boulder, Colo., 1968).

Swanson, N., Codling, K. Excitation of K-shell electrons in Be by soft $x$-ray and 20-keV electrons, J. Opt. Soc. Am. 58, No. 9, 1192-1194 (Sept. 1968).

Tanttila, W. H., Mahler, R. J., James, L. W., Magnetic parametric nuclear spin saturation, Appl. Phys. Letters 13, No. 1, 27-29 (July 1, 1968).

Tauber, S. J., Imprecision-problems for information processing, Am. Doc. 19, No. 4, 413-414 (Oct. 1968).

Thomas, R. N., Symposium on Wolf-Rayet stars: A summary, Astrophysical Letters 2, 147-148 (1968).

Toots, J., Fowler, H. A., Marton, L., Reflectance and $1 / \epsilon$ resonance of beryllium in the far ultraviolet, Phys. Rev. 172, No. 3, 670-676 (Aug. 15, 1968).
Turgel, R. S., A comparator for thermal ac-dc transfer standards, ISA Trans. 6, No. 4, 286-292 (1967).

Van Blerkom, D., Hummer, D. G., A non-LTE theory of overlapping lines near the series limit, Astrophys. J. 154, 741-750 (Nov. 1968).

West, E. D., Churney, K. L., A two-body model for the calorimeters with constant-temperature environment, J. Appl. Phys. 39, No. 9, 4206-4215 (Aug. 1968).

Wiederhorn, S. M., Moisture assisted crack growth in ceramics, Intern. J. Fracture Mech. 4, No. 2, 171-177 (June 1968).

Wildhack, W. A., Improving measurement accuracy for high systems reliability, Electronics 41, No. 5, 280 (Mar. 4, 1968).

Wildhack, W. A., Mason, H. L., Powers, R. S., Jr., Accuracy charts for RF measurements, Proc. IEEE 55, No. 6, 1056-1063 (June 1967).

Wolcott, N. M., Falge, R. L., Jr., Ferromagnetism of CrBe $\mathrm{C}_{12}$, Phys. Rev. 171 , No. 2, 591-595 (July 10, 1968).

Wolcott, N. M., Falge, R. L., Jr., Bennett, L. H., NMR studies in paramagnetic and ferromagnetic $\mathrm{CrBe}_{12}$, Phys. Rev. Letters 21 , No. 8, 546-549 (Aug. 19, 1968).

Zalubas, R., Present state of analysis of the first spectrum of thorium (Th I), J. Opt. Soc. Am. 58, No. 9, 1195-1199 (Sept. 1968).

* Publications for which a price is indicated are available by purchase from the Superintendent of Documents, U.S. Government Printing Office, Washington, D.C. 20402 (foreign postage, one-fourth additional). The NBS non-periodical series are also available from the Clearinghouse for Federal Scientific and Technical Information, Springfield, Va. 22151. Reprints from outside journals and the NBS Journal of Research may often be obtained directly from the authors. 